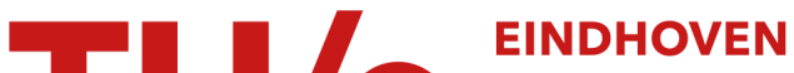 \\ UNIVERSITY OF \\ TECHNOLOGY
}

\section{Anomalous and dimensional scaling in anisotropic turbulence}

\section{Citation for published version (APA):}

Biferale, L., Daumont, I., Lanotte, A., \& Toschi, F. (2002). Anomalous and dimensional scaling in anisotropic turbulence. Physical Review E - Statistical, Nonlinear, and Soft Matter Physics, 66(5), 056306-1/4. [056306]. https://doi.org/10.1103/PhysRevE.66.056306

DOI:

10.1103/PhysRevE.66.056306

Document status and date:

Published: 01/01/2002

\section{Document Version:}

Publisher's PDF, also known as Version of Record (includes final page, issue and volume numbers)

\section{Please check the document version of this publication:}

- A submitted manuscript is the version of the article upon submission and before peer-review. There can be important differences between the submitted version and the official published version of record. People interested in the research are advised to contact the author for the final version of the publication, or visit the $\mathrm{DOI}$ to the publisher's website.

- The final author version and the galley proof are versions of the publication after peer review.

- The final published version features the final layout of the paper including the volume, issue and page numbers.

Link to publication

\section{General rights}

Copyright and moral rights for the publications made accessible in the public portal are retained by the authors and/or other copyright owners and it is a condition of accessing publications that users recognise and abide by the legal requirements associated with these rights.

- Users may download and print one copy of any publication from the public portal for the purpose of private study or research.

- You may not further distribute the material or use it for any profit-making activity or commercial gain

- You may freely distribute the URL identifying the publication in the public portal.

If the publication is distributed under the terms of Article 25fa of the Dutch Copyright Act, indicated by the "Taverne" license above, please follow below link for the End User Agreement:

www.tue.nl/taverne

Take down policy

If you believe that this document breaches copyright please contact us at:

openaccess@tue.nl

providing details and we will investigate your claim. 


\title{
Anomalous and dimensional scaling in anisotropic turbulence
}

\author{
L. Biferale, ${ }^{1,4}$ I. Daumont, ${ }^{1}$ A. Lanotte,${ }^{2,4}$ and F. Toschi ${ }^{3,4}$ \\ ${ }^{1}$ Dipartimento di Fisica, Università “Tor Vergata," Via della Ricerca Scientifica 1, I-00133 Roma, Italy \\ ${ }^{2}$ CNR, ISAC - Sezione di Lecce, Strada Provinciale Lecce-Monteroni Km 1.200, I-73100 Lecce, Italy \\ ${ }^{3}$ CNR, Istituto per le Applicazioni del Calcolo, Viale del Policlinico 137, I-00161 Roma, Italy \\ ${ }^{4}$ INFM, Unità di Tor Vergata, Via della Ricerca Scientifica 1, I-00133 Roma, Italy \\ (Received 6 March 2002; revised manuscript received 9 August 2002; published 20 November 2002)
}

\begin{abstract}
We present a numerical study of anisotropic statistical fluctuations in homogeneous turbulent flows. We give a new argument to predict the dimensional scaling exponents, $\zeta_{d}^{j}(p)=(p+j) / 3$, for the projections of the $p$ th order structure function in the $j$ th sector of the rotational group. We show that the measured exponents are anomalous, showing a clear deviation from the dimensional prediction. Dimensional scaling is subleading and connected to the dynamical fluctuations without phase correlations. Universality of the observed anomalous scaling is discussed both theoretically and by means of numerical simulations at different Reynolds numbers and with different forcing.
\end{abstract}

DOI: 10.1103/PhysRevE.66.056306

PACS number(s): 47.27.Ak, 47.10.+g, 47.27.Eq

In recent years a huge amount of theoretical, numerical, and experimental work has been done in order to study anisotropic turbulent fluctuations [1-11]. Typical questions go from the theoretical point of calculating and measuring anomalous scaling exponents in anisotropic sectors [3-6,10], to the more applied problem of quantifying the rate of recovery of isotropy at small scales $[1,2,7]$. Another important issue is the universality of anisotropic scaling exponents, i.e., whether they are an intrinsic characteristic of the NavierStokes nonlinear evolution or they are fixed by the external anisotropic forcing.

Important steps forward in the analysis of anisotropic fluctuations have been done in Kraichnan models, i.e., passive scalars or vectors advected by isotropic, Gaussian, and $\delta$-correlated in time velocity fields [12], with a large-scale anisotropic forcing [13-15]. In those models, anomalous scaling arises as the result of a nontrivial null-space structure for the advecting operator (zero modes). Also, correlation functions in different sectors of the rotational group show different scaling properties. Scaling exponents are universal: they do not depend on the actual value of the forcing and boundary conditions, and they are fully characterized by the order of the anisotropy. Nonuniversal effects are felt only in coefficients multiplying the power laws.

Similar problems, such as the existence of scaling laws in anisotropic sectors and, if any, the values of the corresponding scaling exponents are at the forefront of the experimental, numerical, and theoretical research for real turbulent flows. Only a few indirect experimental investigations of scaling in different sectors $[4,5]$ and direct decomposition in numerical simulations $[6-8,10]$ have been attempted up to now. On a theoretical ground, the potentiality of $\mathrm{SO}(3)$ decomposition to quantify different degrees of anisotropy for any correlation function has been highlighted only recently [3]. Preliminary experimental evidences of the existence of a scaling law also in sectors with total angular momentum $j$ $=2$ have been reported $[4,5]$. The value of the exponent for the second-order correlation function being close to the dimensional estimate $\zeta_{d}^{j=2}(2)=4 / 3[16]$.
Theoretically speaking, there exists only one previous attempt to give an estimate for scaling exponents of the second order moment and in all sectors [9]. In Ref. [9], a mean-field prediction was formulated for anisotropic scaling of secondorder structure functions obtained by changing the analytical properties of the external forcing. In this paper we provide two different results on this important topic. First, we present a "dimensional" argument able to predict the scaling properties of any moment in any anisotropic sector on the basis of a "local" matching between nonlinear and shear-induced terms in the inertial range. Such a dimensional prediction should play the same role played by Kolmogorov 1941 theory for isotropic fluctuations, i.e., it fixes the referencebackground scaling we should expect in the anisotropic sectors in absence of intermittent fluctuations. Second we show that, after a phase randomization of velocity Fourier components, structure functions posses a dimensional scaling. This is a clear signature that most of the anisotropic intermittency is brought by a nontrivial phase organization while the background field almost follows the dimensional estimate. Finally, to comment on the universality properties of the anomalous anisotropic scaling, we also present some comparison at changing Reynolds numbers and the large-scale forcing.

We have performed two different sets of numerical simulations with large-scale homogeneous and anisotropic forcing obtained by changing both Reynolds numbers and the anisotropy degree of the forcing. The first simulation (DNS) is a random Kolmogorov flow (RKF). The RKF is fully periodic; the large-scale anisotropic random forcing points in one direction, $\hat{z}$, has a spatial dependency only from the $x$ coordinate and it is different from zero at the two wave numbers: $\quad \boldsymbol{k}_{1}=(1,0,0), \boldsymbol{k}_{2}=(2,0,0) . \quad$ Namely, $\quad \boldsymbol{f}_{i}\left(\boldsymbol{k}_{\{1,2\}}\right)$ $=\delta_{i, 3} f_{\{1,2\}} \exp \left(\theta_{\{1,2\}}\right)$, where $f_{\{1,2\}}$ are fixed amplitudes and $\theta_{\{1,2\}}$ are independent random phases, $\delta$ correlated in time. We simulated the RKF at resolution $128^{3}$ and $256^{3}$ and collected up to 70 eddy turnover times; Reynolds numbers based on the gradient scale are $\operatorname{Re}_{\lambda}=60$ and $\operatorname{Re}_{\lambda}=90$, respectively. Energy is dissipated by a hyperviscosity term at small scales. 
The second set of simulations was forced by fixing the total energy on a subset of Fourier modes falling in a plane $\boldsymbol{k}=\left(k_{x}, k_{y}, 0\right)$ with $|\boldsymbol{k}|<4$. Both simulations are homogeneous and anisotropic, the degree of anisotropy in the latter case is much smaller than in the RKF. Most of the quantitative results will be discussed for the RKF. The second set of simulations is used to discuss the degree of universality of anisotropic scaling.

Anisotropy is studied by means of $\mathrm{SO}(3)$ decomposition of longitudinal structure functions, $S_{p}(\boldsymbol{R})=\langle\{[\boldsymbol{v}(\boldsymbol{x}+\boldsymbol{R})$

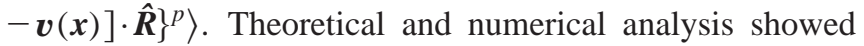
$[3,6,10]$ that one must first decompose the structure functions onto irreducible representations of the rotational group and then study the scaling behavior of the projections. In practice, being the longitudinal structure functions scalar objects, their decomposition reduces to the projections on the spherical harmonics,

$$
S_{p}(\boldsymbol{R})=\sum_{j=0}^{\infty} \sum_{m=-j}^{j} S_{p}^{j m}(|\boldsymbol{R}|) Y_{j m}(\hat{\boldsymbol{R}}) .
$$

As usual, we use indexes $(j, m)$ to label, respectively, the total angular momentum and its projection along a reference axis, say $\hat{z}$. The whole physical information is brought by the projections $S_{p}^{j m}(R)$. In particular, the main question we want to address here concerns their scaling properties,

$$
S_{p}^{j m}(|\boldsymbol{R}|) \sim A_{j m}|\boldsymbol{R}|^{\zeta^{j, m}(p)} .
$$

First, we need an estimate for the dimensional values of the exponents $\zeta_{d}^{j, m}(p)$ in all sectors. Our argument is based on the idea that large-scale energy pumping and/or boundary conditions are such as to enforce a large-scale anisotropic driving velocity field $\boldsymbol{U}$. A prediction for intermediate (small) scales anisotropic fluctuations may then be obtained by studying the influence of the large scale $\boldsymbol{U}$ on the inertial range. By decomposing the velocity field in a small scale component, $\boldsymbol{u}$, and a large-scale anisotropic component $\boldsymbol{U}$ one finds the following equation for the time evolution of $\boldsymbol{u}$ :

$$
\partial_{t} u_{i}+u_{k} \partial_{k} u_{i}+U_{k} \partial_{k} u_{i}+u_{k} \partial_{k} U_{i}=-\partial_{i} p+\nu \Delta u_{i} .
$$

The major effect of the large-scale field is given by the instantaneous shear $\mathcal{S}_{i k}=\partial_{k} U_{i}$ which acts as an anisotropic forcing term on small scales.

A matching argument can be built as follows. Let us first consider the equation of motion for two-point quantities $\left\langle u_{l}\left(\boldsymbol{x}^{\prime}\right) u_{i}(\boldsymbol{x})\right\rangle$ in the stationary regime. We may balance inertial terms and shear-induced terms as follows:

$$
\left\langle u_{l}\left(\boldsymbol{x}^{\prime}\right) u_{k}(\boldsymbol{x}) \partial_{k} u_{i}(\boldsymbol{x})\right\rangle \sim\left\langle\mathcal{S}_{i k}(\boldsymbol{x}) u_{l}\left(\boldsymbol{x}^{\prime}\right) u_{k}(\boldsymbol{x})\right\rangle,
$$

which allows for a dimensional estimate of the anisotropic components of the left-hand side (LHS) in terms of the righthand side (RHS) shear intensity and of the $\langle u u\rangle$ isotropic part.

Similarly for three-point quantities we have (neglecting here, for simplicity, tensorial notation): $\langle и и и д и\rangle \sim\langle\mathcal{S} и и и\rangle$ which can be easily generalized to any order velocity corre- lations. The shear term is a large-scale "slow" quantity and therefore, as far as scaling properties are concerned, we may safely estimate: $\left\langle\mathcal{S}_{i k}(\boldsymbol{x}) u_{l}\left(\boldsymbol{x}^{\prime}\right) u_{k}(\boldsymbol{x})\right\rangle \sim D_{i k}\left\langle u_{l}\left(\boldsymbol{x}^{\prime}\right) u_{k}(\boldsymbol{x})\right\rangle$. Here the matrix $D_{i k}$ is associated to the combined probability to have a given shear and a given small scale velocity configuration. Clearly the $D_{i k}$ tensor brings angular momentum only up to $j=2$. One may therefore argue, by using simple composition of angular momenta, the following dimensional matching [17]:

$$
S_{p}^{j}(R) \sim R \mathcal{S} S_{p-1}^{j-2}(R),
$$

where $S_{p}^{j}(R)$ is the projection on the $j$ th sector of the $p$ th order correlation function at scale $R$ [see Eq. (1)], and $\mathcal{S}$ is the intensity of the shear term $D_{i k}$ in the $j=2$ sector. For instance, the leading behavior of the $j=2$ anisotropic sector of the third-order correlation function in the LHS of Eq. (5) is given by the coupling between the $j=2$ components of $D_{i k}$ and the $j=0$ sector of the second-order velocity correlation in the RHS of Eq. (5): $S_{3}^{2}(R) \sim R S S_{2}^{0}(R) \sim R^{\xi_{d}^{j=2}(3)}$. By using the same argument and considering that now we know the scaling of $j=0$ and $j=2$ sectors of the third-order correlation, we may estimate the scaling exponents of the fourth-order correlation for $j=2,4$. The procedure is easily extended to all orders, leading to the following general expression:

$$
\zeta_{d}^{j}(p)=\frac{(p+j)}{3},
$$

which has been obtained, for simplicity, by neglecting the intermittency effects in the isotropic sector.

In this way, giving as input only the isotropic exponents, $\zeta_{d}^{j=0}(p)$, we are able to predict the scaling exponents up to $j=2$ for the third-order structure functions, to $j=4$ for the fourth order, to $j=6$ for the fifth order, and so on. We may do a little better by giving a prediction also for anisotropic fluctuations of second-order correlation functions. This cannot be simply obtained by using the equations of motion, because the first one involving velocity correlations at different spatial locations, i.e., inertial range quantities, is that for $\partial_{t}\left\langle u_{i}(x) u_{j}\left(x^{\prime}\right)\right\rangle$, which fixes a constraint only for the thirdorder correlation function (4). A way out is to ask the second-order anisotropic fluctuations to be analytic in the shear intensity $\mathcal{S}$ consistently with what one finds for higherorder structure functions by the above dimensional estimate. With this assumption, we recover for $j=2$ Lumley prediction [16], $\zeta_{d}^{j=2}(2)=4 / 3$ by simply writing the first two terms dimensionally consistent with an expansion in the shear intensity: $\langle u u\rangle \sim(\epsilon R)^{2 / 3}+\epsilon^{1 / 3} \mathcal{S} R^{4 / 3}+\cdots$ where the first corresponds to the isotropic scaling, while the second captures anisotropies up to $j=2$ (higher $j$ sectors could be captured by adding other terms in the expansions). By using this argument, we may now remove the limit of validity of the dimensional prediction (6) and extend it to all $p$ values.

Let us notice that prediction (6) must be considered as the systematic generalization of Lumley argument [16] to all structure functions orders and to all SO(3)sectors. To our knowledge, this is the first prediction of scaling properties in 


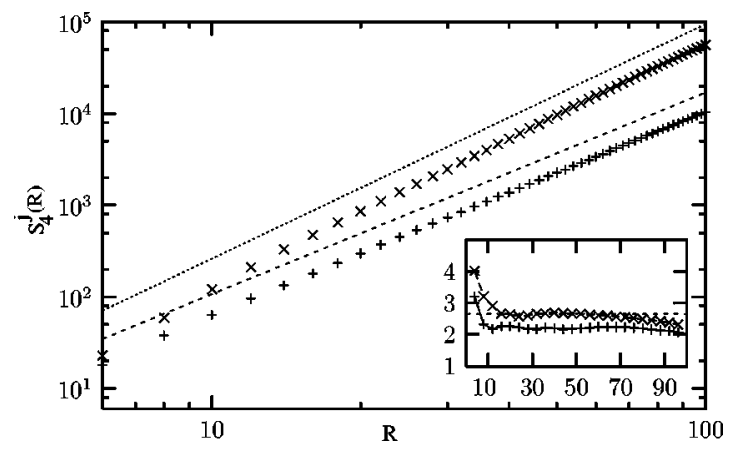

FIG. 1. Comparison of scaling properties before $(+)$ and after $(X)$ phases randomization of the fourth-order structure function for $j=4, m=0$. Straight lines are the best fit slopes in the inertial range. In the inset, the changes for the logarithmic local slopes (same symbols); the horizontal dashed line corresponds to the dimensional prediction $\zeta_{d}^{4}(4)=8 / 3$. Similar results hold for other anisotropic sectors (not shown).

all sectors and for all orders of moments. It is worth to note that at difference with previous attempts made for the case of the second-order structure function [9], here the tensorial nature of the shear-induced terms is taken into account.

We come to our numerical results for the $\mathrm{SO}(3)$ decomposition of longitudinal structure functions. In Ref. [6] a first quantitative analysis of scaling was presented. We repeated and extended the same analysis on a larger ensemble of configurations of the random Kolmogorov flow at two different Reynolds numbers. Now, except for the sector with $j=2$, where a sign oscillation in the projections, $S_{p}^{j, m}(R)$, does not allow a quantitative estimate of exponents, we are able to measure with higher accuracy the scaling exponents up to $j$ $=6$ and $p=6$. All measured exponents show a clear departure from the dimensional prediction. For example, we measure in the $j=4$ sectors the values $\zeta^{4}(2)=1.65(5), \zeta^{4}(4)$ $=2.20(5), \zeta^{4}(6)=2.55(10)$, and in the $j=6$ sector, $\zeta^{6}(2)$ $=3.2(2), \zeta^{6}(4)=3.1(2), \zeta^{6}(6)=3.3(2)$. This is a clear indication that anisotropic scaling exponents are intermittent. Nevertheless, the dimensional prediction (6) plays an important role in fixing the subleading background scaling as it can be demonstrated by looking at those fluctuations which are phase independent.

We have taken the stationary configurations of the RKF and randomly reshuffled all velocity phases: $\hat{u}_{i}(\boldsymbol{k})$ $\rightarrow P_{i l}(\boldsymbol{k}) \hat{u}_{l}(\boldsymbol{k}) \exp \left[i \theta_{l}(\boldsymbol{k})\right]$, where $P_{i l}(\boldsymbol{k})$ is the incompressibility projector. Doing so, we expect to filter out the dominant intermittent fluctuations coming from the inertial evolution, or at least those intermittent contributions connected to nontrivial phase organization. In other words, we expect that once canceled the anomalous fluctuations, the subdominant fluctuations due to the dimensional balancing with the forcing-shear terms will show up. It is worth to remark that after phase randomization the statistics of the velocity field still stays non-Gaussian, because velocity Fourier amplitudes are not changed.

In Fig. 1 we show the results for the decomposition of the fourth-order structure functions (after phase randomization) in the $j=4, m=0$ anisotropic sector. As it can be seen, scal-
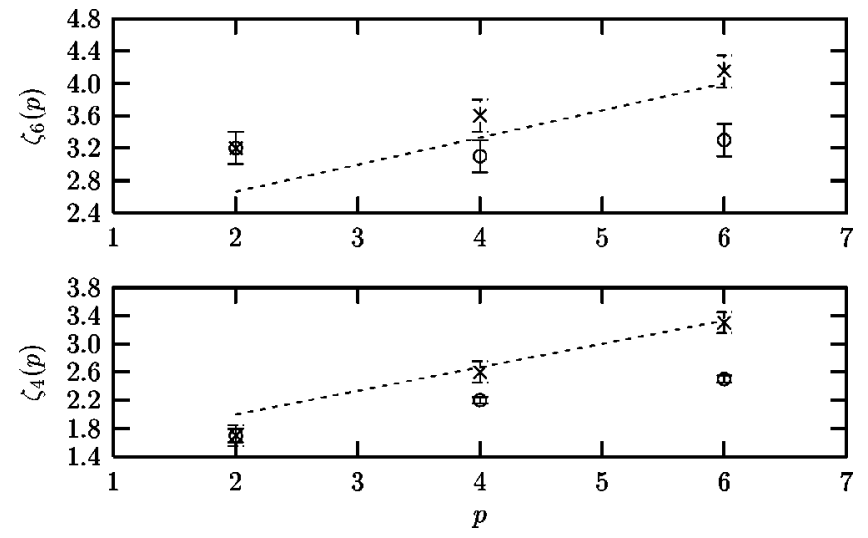

FIG. 2. Comparison between the dimensional estimate, $\zeta_{d}^{j}(p)$ $=(p+j) / 3$ (straight lines), the measured exponents $\zeta^{j}(p)(\bigcirc)$, and the exponents $\zeta_{r}^{j}(p)$ obtained after random dephasing $(\times)$, for $p$ $=2,4,6$. Top, sector $j=6$; bottom, sector $j=4$. Error bars are estimated by looking both at fluctuations in the local slopes and by performing the same analysis on different subsamples.

ing properties change significantly going from the anomalous value (before randomization) to the dimensional prediction (after randomization). This happens for all sectors and moments we have measured, as it is summarized in Fig. 2, with the notable exception of the second-order structure function where phase randomization has almost no effect. This is an interesting fact that can have at least two explanations. Phase randomization is not enough to completely filter out intermittency, especially for two-point quantities which should be less sensible to phase correlation. Or, as noticed before, because second-order correlation function is not constrained by any equation of motion, dimensional scaling may never exist for it even not as a subleading contribution.

The last issue we want to discuss is that of universality of anomalous scaling, i.e., the dependency on the large-scale forcing mechanism.

In Kraichnan models there exists a tight link between the presence of anomalous scaling and its universality, due to the zero-mode mechanism [12]. Unfortunately, in the Navier-

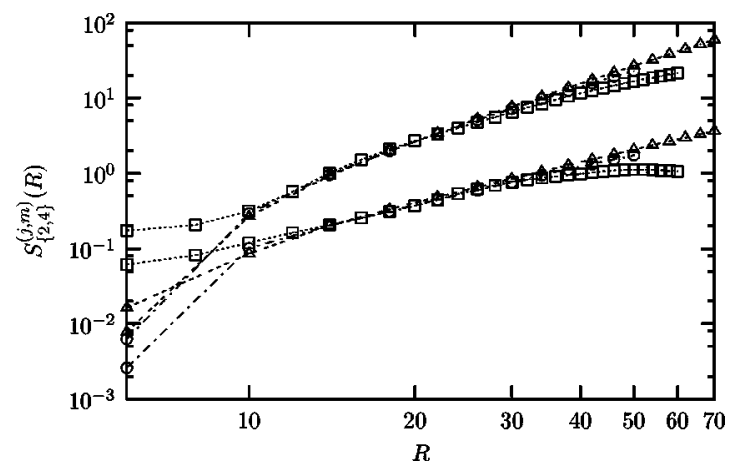

FIG. 3. Log-log plot of the most intense anisotropic sectors for structure functions of order 2 and 4, at changing Reynolds numbers and large-scale forcing. On the top, three curves: $S_{4}^{2,2}(R)$ for the $\mathrm{RKF}$ at $\operatorname{Re}_{\lambda}=60(\bigcirc)$ and $\operatorname{Re}_{\lambda}=90(\triangle)$, and for the second largescale forcing (see text) at $\operatorname{Re}_{\lambda}=90(\square)$. In the bottom, the same but for $S_{2}^{4,0}(R)$. 
Stokes case the linear hierarchy of equations governing the spatial-time evolution of the $n$th point correlation functions is not closed. Inertial solutions with different scaling exponents obtained by changing the large-scale forcing may then exist, while in the Kraichnan case only prefactors of the scaling laws depend on the large-scale forcing. Because of this, it is of primary importance to compare different experiments and numerical simulations in order to understand the degree of universality of the inertial range scaling.

In Fig. (3) we compare the scaling properties of the most important anisotropic contributions for second- and fourthorder structure functions for two different forcings and two different Reynolds numbers.

As it can be seen, despite the anisotropic contributions for the second forcing being much noisier at large scale, we have a quite good qualitative agreement for the scaling properties in the inertial range. This is a first indication that anisotropic scaling might be universal. Clearly, much more extensive tests at higher Reynolds numbers and with different anisotropic forcings have to be performed before drawing any firm conclusion on the issue. In conclusions we have presented a dimensional argument able to predict scaling exponents for all structure functions in any anisotropic sector. We have shown by DNS that anisotropic scaling exponents deviate from the previous dimensional prediction, showing anomalous values. When performing a random reshuffling of all velocity phases, the dimensional scaling, otherwise subleading, shows up. The present work is intended to give qualitative indications about anisotropic anomalous fluctuations when varying the forcing and the Reynolds number.

We thank I. Arad, M. Cencini, and M. Vergassola for useful comments. This research was supported in part by the EU under Grant No. HPRN-CT 2000-00162, "Non Ideal Turbulence" and by the INFM (Iniziativa di Calcolo Parallelo).
[1] A. Pumir and B. Shraiman, Phys. Rev. Lett. 75, 3114 (1995).

[2] S. Garg and Z. Warhaft, Phys. Fluids 10, 662 (1998).

[3] I. Arad, V.S. L'vov, and I. Procaccia, Phys. Rev. E 59, 6753 (1999).

[4] I. Arad, B. Dhruva, S. Kurien, V.S. L'vov, I. Procaccia, and K.R. Sreenivasan, Phys. Rev. Lett. 81, 5330 (1998).

[5] S. Kurien and K.R. Sreenivasan, Phys. Rev. E 62, 2206 (2000).

[6] L. Biferale and F. Toschi, Phys. Rev. Lett. 86, 4831 (2001).

[7] L. Biferale and M. Vergassola, Phys. Fluids 13, 2139 (2001).

[8] L. Biferale, D. Lohse, I.M. Mazzitelli, and F. Toschi, J. Fluid Mech. 452, 39 (2002).

[9] S. Grossmann, A. Von der Heydt, and D. Lohse, J. Fluid Mech. 440, 381 (2001).

[10] I. Arad, L. Biferale, I. Mazzitelli, and I. Procaccia, Phys. Rev.
Lett. 82, 5040 (1999).

[11] X. Shen and Z. Warhaft, Phys. Fluids 14, 370 (2002)

[12] G. Falkovich, K. Gawȩdzki, and M. Vergassola, Rev. Mod. Phys. 73, 913 (2001).

[13] A. Lanotte and A. Mazzino, Phys. Rev. E 60, R3483 (1999).

[14] I. Arad, L. Biferale, and I. Procaccia, Phys. Rev. E 61, 2654 (2000).

[15] I. Arad, V.S. L'vov, E. Podivilov, and I. Procaccia, Phys. Rev. E 62, 4904 (2000).

[16] J.L. Lumley, Phys. Fluids 8, 1056 (1967).

[17] From now on, for the sake of simplicity, we neglect the dependency from the projection of the total angular momentum on the $z$ axis, associated to the $m$ index. 